\title{
DIDS blocks a chloride-dependent current that is mediated by the 2B protein of enterovirus 71
}

Cell Research (2011) 21:1271-1275. doi:10.1038/cr.2011.112; published online 12 July 2011

\section{Dear Editor,}

Enterovirus 71 (EV71), which belongs to the genus Enterovirus of the family Picornaviridae, is one of the major pathogens that cause hand, foot and mouth disease, primarily in infants and young children. In recent years, epidemic and sporadic outbreaks of neurovirulent EV71 infections have been reported in many countries and regions, including Japan, China and Taiwan [1]. However, there is still no effective antiviral treatment against severe EV71 infections, and no vaccine is available.

During their life cycle, viruses can induce many changes in their host cells including increased plasma membrane permeability [2]. Although its detailed mechanism remains largely unknown, this effect on the plasma membrane could be due to the expression of many virusencoded proteins [3-5]. The expression of protein $2 \mathrm{~B}$ from polioviruses and coxsackieviruses can increase the permeability of the cell membrane to the translational inhibitor hygromycin $B[6,7]$. A recent study that focused on protein $2 \mathrm{~B}$ from coxsackievirus $\mathrm{B} 3$ (CVB3) showed that $2 \mathrm{~B}$ can facilitate virus release by increasing the concentration of free cytosolic $\mathrm{Ca}^{2+}[8]$. However, little is known about $2 \mathrm{~B}$ proteins from other enteroviruses, and the detailed mechanisms of how $2 \mathrm{~B}$ changes cellular ion homeostasis and, thus, promotes virion release remain unclear.

Because the 2B protein of CVB3 can form homodimers and homotetramers that are located primarily in the cell membrane system, including the Golgi complex [9, $10]$, we asked whether the $2 \mathrm{~B}$ proteins of EV71 might share similar characteristics. To address this question, the sequences of the $2 \mathrm{~B}$ genes from these two viruses were compared. These sequences have relatively high similarity, especially within their two transmembrane domains (TM1 and TM2), structures that are essential for the function of viroporins (Supplementary information, Figure S1A). Next, we examined the subcellular localization of the EV71 2B protein in human rhabdomyosarcoma (RD) cells and found that it colocalized with the Golgi complex (Supplementary information, Figure S1B).
Finally, when the 2B protein was fused to an HA tag and transiently overexpressed in $293 \mathrm{~T}$ cells, a $\sim 13-\mathrm{kDa}$ monomer, a putative $26-\mathrm{kDa}$ dimer and a putative $52-$ $\mathrm{kDa}$ tetramer were detected after pull down in an immunoprecipitation assay using an anti-HA antibody. After treatment with $\beta$-mercaptoethanol ( $\beta$-ME), which reduces disulfide bonds within proteins, the putative dimer and tetramer bands were no longer detected (Supplementary information, Figure S1C). These data indicate that the 2B protein in EV71 has characteristics in common with ion channels.

To test whether the EV71 2B protein indeed forms an ion channel, we used two-electrode voltage-clamp recordings to measure membrane currents in Xenopus oocytes that were injected with complementary RNA (cRNA) encoding 2B protein with a C-terminal HA tag. Expression of 2B-HA in oocytes was confirmed by immunostaining with an anti-HA antibody. Using confocal microscopy, the $2 \mathrm{~B}-\mathrm{HA}$ protein was shown to mainly localize at the cell membrane, suggesting that $2 \mathrm{~B}$ is an integral membrane protein (Supplementary information, Figure S2). Thirty-six to forty-eight hours after injection, a significant increase in membrane conductance was detected in the oocytes (Figure 1Ai and Aii). We also generated a dysfunctional mutant in which the entire TM1 domain was deleted. When injected into oocytes, this mutant showed a dramatic decrease in current, indicating that the current is not induced simply by RNA injection (Figure 1Aiii). An $I-V$ curve generated from four oocytes that were injected with $25 \mathrm{ng}$ of cRNA showed that the $2 \mathrm{~B}$ protein induced a current that was $\sim 4000 \mathrm{nA}$ at -150 $\mathrm{mV}$ and the reversal potential is around $-30 \mathrm{mV}$ (Figure 1B). These results suggest that the $2 \mathrm{~B}$ protein might function as an ion channel in the oocyte membrane, thereby generating the observed current.

To explore the ion selectivity of the 2B-mediated current, ion replacement experiments were performed. Both the inward and outward currents decreased when $\mathrm{NaCl}$ was replaced with sodium gluconate (Figure 1Aiv and $1 \mathrm{C})$, and the reversal potential shifted from approximately $-30 \mathrm{mV}$ to $-10 \mathrm{mV}$. In contrast, replacing the ex- 
A
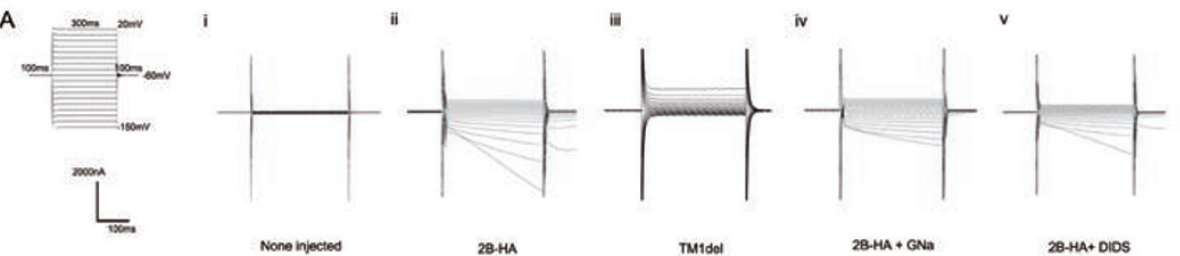

None now

$28+$ HA

TMidel

$28-+4+$ GNa

2B-HA+DIOS

B

$2000+$

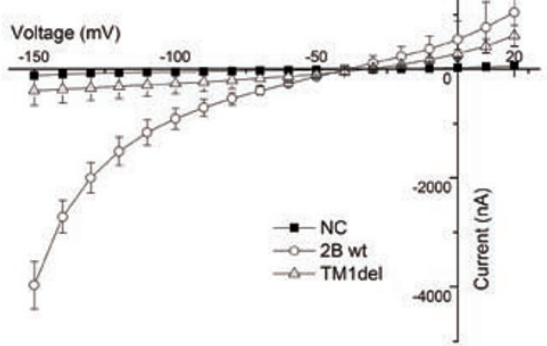

C

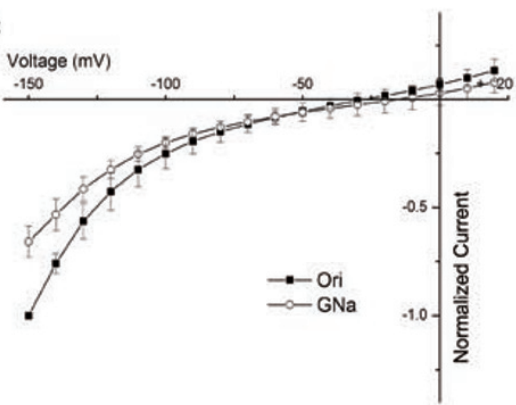

D

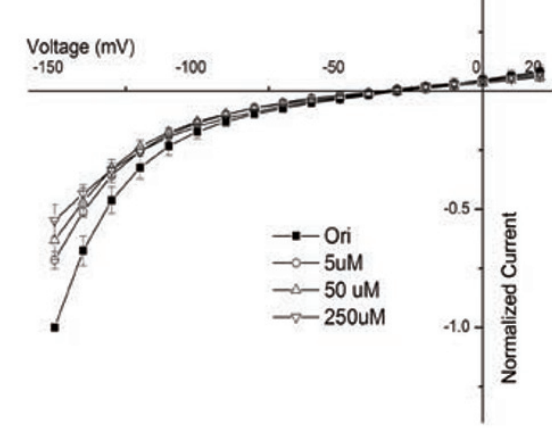

E

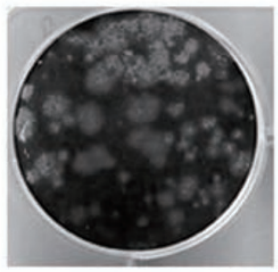

No Drug

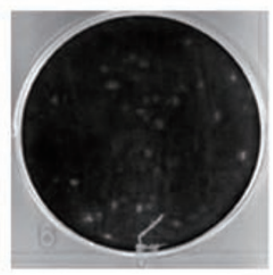

DIDS treated
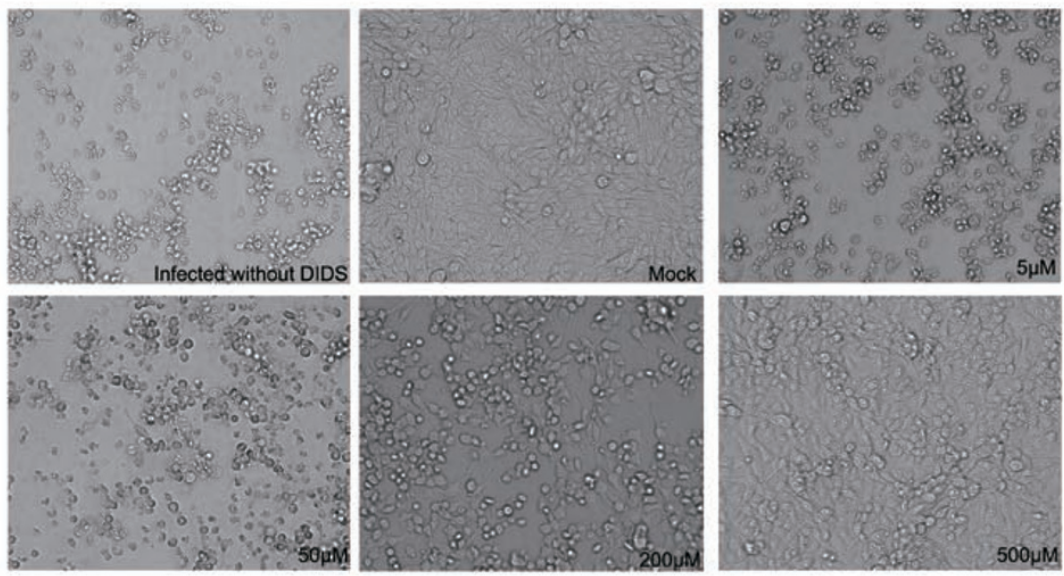

G
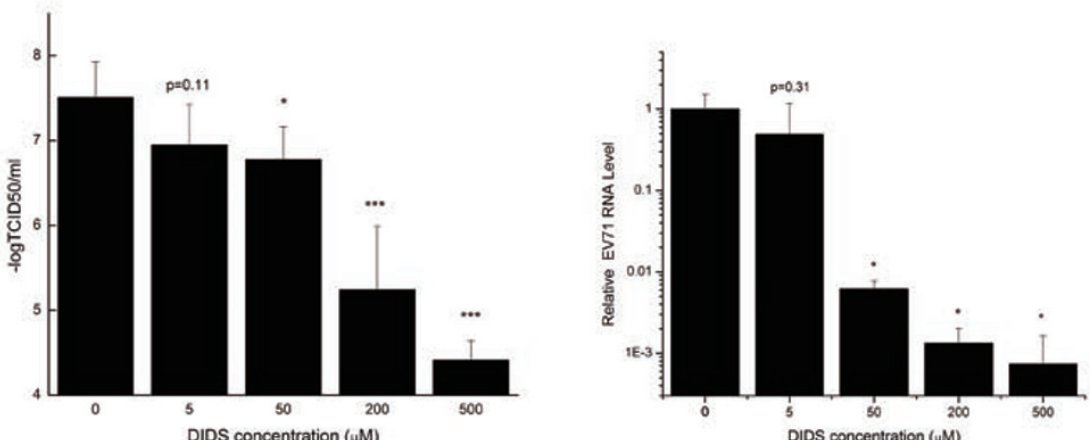

Cell Research | Vol 21 No 8 | August 2011 
ternal potassium or sodium with $\mathrm{N}$-methyl-D-glucamine only had a slight effect on the current (data not shown). Together, these data led to the hypothesis that chloride is the principal ion carried in the $2 \mathrm{~B}$-mediated current.

To further test whether $2 \mathrm{~B}$-mediated conductance is carried by chloride, we examined the effect of chloride channel inhibitors. The 2B-mediated current was partially inhibited by 4,4'-diisothiocyano-2,2'-stilbenedisulfonic acid (DIDS; Figure 1Av). This inhibitory effect was concentration dependent (Figure 1D) but was difficult to recover. (We washed the oocyte with standard bath solution for about $3 \mathrm{~min}$ to attempt recovery.) Taken together, these data indicate that the $2 \mathrm{~B}$ protein induces a chloridedependent current that can be partially blocked by DIDS.

Finally, we tested whether DIDS can affect virus life cycle in a cell culture system. A plaque assay revealed that even a relatively low concentration $(10 \mu \mathrm{M})$ of DIDS significantly inhibits virus production, as the plaque size was dramatically reduced after treatment (Figure 1E). Next, RD cells were infected with the EV71 virus, followed by the replacement of fresh medium containing DIDS 30 min later. Forty-eight hours later, an EV71-induced cytopathic effect (CPE) was observed microscopically, and this effect was significantly smaller in the DIDS-treated cells compared with the control cells. The inhibition of CPE by DIDS was concentration dependent, with a range of 5-500 $\mu \mathrm{M}$ (Figure 1F). A volume of $500 \mu \mathrm{M}$ DIDS conferred the maximum protective effect against CPE. Higher concentrations (800 and $1000 \mu \mathrm{M}$, data not shown) were tested; however, as was seen for the $50 \%$ tissue culture infective dose $\left(\mathrm{TCID}_{50}\right)$, these higher concentrations did not cause significantly more inhibition than $500 \mu \mathrm{M}$. Moreover, at these higher concentrations, DIDS exerted morphological effects on the cells. In addition, we collected the cell supernatant for $\mathrm{TCID}_{50}$ (Figure $1 \mathrm{G}$, left panel) and real-time RT-PCR
(Figure 1G, right panel) analyses. The results show that both the viral titer and the RNA levels in the supernatant markedly decreased after DIDS treatment, and this effect was concentration dependent. In conclusion, DIDS appears to protect RD cells from EV71 viral infection by inhibiting virus production.

Many viruses encode small hydrophobic proteins that form a pore-like structure and increase the membrane permeability of cells. These proteins were initially described as viroporins and are generally considered to be promising targets for antiviral drugs. In this study, we observed a similarity between the sequences of the $2 \mathrm{~B}$ proteins from EV71 and CVB3, and then investigated their potential function as ion channels in Xenopus oocytes. Because they express low levels of endogenous ion channels, oocytes are a good system for testing the putative ion channels properties of proteins. However, we observed that the subcellular localization of $2 \mathrm{~B}$ is different in oocytes compared with RD cells. This may be due to different expression patterns in these two systems. Moreover, it has been reported that the localization of $2 \mathrm{~B}$ proteins is different when they are coexpressed with other viral products rather than expressed alone [11]. Therefore, the putative ion channel function and the native localization of the $2 \mathrm{~B}$ protein should be detected simultaneously in an infection-permeability coupled assay.

A recent study has suggested that the CVB3 $2 \mathrm{~B}$ protein can mediate cytosolic calcium elevation [8]. However, our data demonstrate that the current induced by the EV71 2B protein is mainly carried by chloride ions. In this regard, the calcium elevation in the cytoplasm is likely to be an indirect phenomenon induced by the $2 \mathrm{~B}$ protein. Some investigators have reported that certain anion channels in the Golgi complex may serve as the source of counter anions for $\mathrm{H}^{+}$-ATP transporters [12]. Moreover, the calcium uptake mechanism requires ATP

Figure 1 (A) The general protocol used in the voltage-clamp experiments is shown in the left panel. (i, ii) At 24 to $36 \mathrm{~h}$ after cRNA injection, currents were recorded using a two-electrode voltage clamp, and injected and non-injected oocytes were compared. (iii) Raw current in an oocyte expressing the dysfunctional mutant TM1del. (iv) Raw current in an oocyte expressing 2B$\mathrm{HA}$ after the extracellular $\mathrm{NaCl}$ in the oocyte Ringer solution (ORi) was replaced with equimolar sodium gluconate (GNa). (v) Raw current in an oocyte expressing 2B-HA and treated with $250 \mu \mathrm{M} 4,4^{\prime}$-diisothiocyano-2,2'-stilbenedisulfonic acid (DIDS). (B) Current-voltage $(I-V)$ curves of the 2B-HA-mediated currents. The normalized currents from the chloride replacement experiment are plotted in $\mathbf{C}$ as the current at each voltage normalized to the peak current at $-150 \mathrm{mV}$. The corresponding normalized currents following the application of a given dose of DIDS are shown in $\mathbf{D}$. The data in B-D are presented as mean \pm SD and were generated from at least three separate experiments. (E) A plaque assay was performed in cells treated with or without $10 \mu \mathrm{M}$ DIDS. (F) RD cells were mock infected or infected with EV71 at an MOI of 0.002 and then cultured in medium containing the indicated concentration of DIDS for an additional $48 \mathrm{~h}$. The cytopathic effect (CPE) was observed using microscopy (at $100 \times$ magnification). (G) RD cells were infected with the EV71 virus at an MOI of 0.002 for 30 min, and cultured in fresh medium containing indicated concentration of inhibitor. Two days after infection, the supernatant was collected for tissue culture infective dose $\left(T_{C} I_{50}\right)$ analysis (left panel) or real-time RT-PCR analysis (right panel). The data are presented as mean \pm SD and were generated from at least three separate experiments. $\left({ }^{\star} P<0.05\right.$, ${ }^{* \star \star} P<0.001$, compared with the no DIDS group) 
to maintain homeostasis in the Golgi complex [13]. Thus, the 2B-mediated, chloride-dependent current may perturb anion homeostasis in the Golgi complex and ultimately lead to calcium leakage into the cytoplasm. Nevertheless, whether or not calcium ions can directly pass through a 2B channel needs more investigations. It remains unclear how $2 \mathrm{~B}$ is involved in the viral life cycle. As a cytolytic virus, EV71 may primarily release its progeny via cell lysis. The 2B expression would perturb ion homeostasis and may disrupt cellular function [8]. Some studies have suggested that $2 \mathrm{~B}$ induces cell apoptosis via a caspasedependent pathway [14], which may explain the role of 2B in cell lysis. However, little direct evidence has been provided to establish a link between changes in permeability and 2B-induced apoptosis.

In this study, DIDS potently inhibited virus production in RD cells. We hypothesize that DIDS suppresses virus release by inhibiting the $2 \mathrm{~B}$ protein-mediated chloridedependent current. However, as a classic anion exchanger blocker, DIDS also has effects on the cells themselves [15]. Nevertheless, in our study, DIDS had no significant effect on either cell growth or morphology at relatively low concentrations $(\leq 500 \mu \mathrm{M})$, suggesting that the inhibition of DIDS on EV71 production can be primarily attributed to its effects on the 2B-mediated current. Clearly, additional experiments are needed to further test this hypothesis.

In summary, we report that the $2 \mathrm{~B}$ protein may mediate a chloride-dependent current in oocytes, and DIDS, an inhibitor of this current, blocks virus production and virus-induced CPE in RD cells. This study provides a new approach for identifying potential anti-EV71 drugs. Moreover, understanding the properties of the $2 \mathrm{~B}$ protein as an ion channel may help shed light on the life cycle of this virus.

\section{Acknowledgments}

We thank Prof FJ van Kuppeveld (Radboud University Nijmegen Medical Centre) for useful discussions and Dr Jun-ichi Miyazaki (Osaka University) for providing the pCAGGS plasmid. We also thank Dr Sheri Skinner for English editing. This work was supported by a CAS project (KSCX2-YW-R-161), a grant from the National Ministry of Science and Technology (20072714), grants from the National Natural Science Foundation of China (30950002, 30623003, 30721065,30801011, 30870126 and 90713044), grants from the Science and Technology Commission of Shanghai Municipality (08DZ2291703, 088014199 and 08431903004), a National Science and Technology Major Project (2008ZX10002014, 2008ZX10004-002 and 2009ZX10004-105), a grant from the National 973 Key Project (2007CB512404), grants from SPHRF (SPHRF2008001 and SPHRF2009001), a grant from the National 863 Project (2006AA02A247) and a grant from the Li Kha Shing Foundation.
Shiqi Xie ${ }^{1, *}$, Kai Wang ${ }^{1, *}$, Wenjing $\mathrm{Yu}^{1}$, Wei $\mathrm{Lu}^{1,8}$,

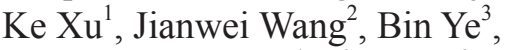
Wolfgang Schwarz ${ }^{4,5}$, Qi Jin ${ }^{2}$, Bing Sun ${ }^{1,7}$

${ }^{I}$ Key Laboratory of Molecular Virology and Immunology, Institut Pasteur of Shanghai, Shanghai Institutes for Biological Sciences, Chinese Academy of Sciences, 225 South Chongqing Road, Shanghai 200025, China, ${ }^{2}$ Institute of Pathogen Biology, Chinese Academy of Medical Sciences. Beijing 100176, China; ${ }^{3}$ Department of Medicine, Section of Cardiovascular Medicine, School of Medicine, Room 24, SMI building, 1300 University Avenue, University of Wisconsin, Madison, WI 53706, USA, ${ }^{4}$ Max Planck Guest Laboratory, Shanghai Institutes for Biological Sciences, Chinese Academy of Sciences, 320 Yueyang Road, Shanghai 200031, China; ${ }^{5}$ Max Planck Institute for Biophysics, Max-von-Laue-Strasse 3, 60438 Frankfurt/M, Germany; ${ }^{6}$ Shanghai Research Center for Acupuncture and Meridians, 199 Guo Shoujing Road, Shanghai 201203, China; ${ }^{7}$ Laboratory of Molecular Cell Biology, Institute of Biochemistry and Cell Biology, Shanghai Institutes for Biological Sciences, Chinese Academy of Sciences, 320 Yueyang Road, Shanghai 200031, China

*These two authors contributed equally to this work.

Correspondence: Bing Sun ${ }^{\mathrm{a}}$, Qi Jin ${ }^{\mathrm{b}}$

${ }^{\mathrm{a}}$ Tel: +86-21-63851929

E-mail: bsun@sibs.ac.cn

'Tel: +86-10-67876915

E-mail: zdsys@vip.sina.com

${ }^{8}$ Current address: Molecular Development Section, Laboratory of Immunology, National Institute of Allergy and Infectious Diseases, National Institutes of Health, Bethesda, MD 20892, USA

\section{References}

1 Weng KF, Chen LL, Huang PN, Shih SR. Neural pathogenesis of enterovirus 71 infection. Microbes Infect 2010; 12:505-510.

2 Carrasco L. Modification of membrane permeability by animal viruses. Adv Virus Res 1995; 45:61-112.

3 Gonzalez ME, Carrasco L. Viroporins. FEBS Lett 2003; 552:28-34.

4 Fischer WB, Kruger J. Viral channel-forming proteins. Int Rev Cell Mol Biol 2009; 275:35-63.

5 Wang K, Xie S, Sun B. Viral proteins function as ion channels. Biochim Biophys Acta 2011; 1808:510-515.

6 Doedens JR, Kirkegaard K. Inhibition of cellular protein secretion by poliovirus proteins $2 \mathrm{~B}$ and 3A. EMBO J 1995; 14:894907.

7 van Kuppeveld FJ, Melchers WJ, Kirkgaard K, Doedens JR. Structure-function analysis of coxsackie B3 virus protein $2 \mathrm{~B}$. Virology 1997; 227:111-118.

8 van Kuppeveld FJ, Hoenderop JG, Smeets RL, et al. Coxsackievirus protein 2B modifies endoplasmic reticulum membrane and plasma membrane permeability and facilitates virus release. EMBO J 1997; 16:3519-3532.

9 de Jong AS, Melchers WJ, Glaudemans DH, Willems PH, van Kuppeveld FJ. Mutational analysis of different regions in 
the coxsackievirus 2B protein: requirements for homo-multimerization, membrane permeabilization, subcellular localization, and virus replication. J Biol Chem 2004; 279:1992419935.

10 de Jong AS, Wessels E, Dijkman HB, et al. Determinants for membrane association and permeabilization of the coxsackievirus 2B protein and the identification of the Golgi complex as the target organelle. J Biol Chem 2003; 278:1012-1021.

11 Suhy DA, Giddings TH, Kirkegaard K. Remodeling the endoplasmic reticulum by poliovirus infection and by individual viral proteins: an autophagy-like origin for virus-induced vesicles. J Virol 2000; 74:8953-8965.

12 Nordeen MH, Jones SM, Howell KE, Caldwell JH. GOLAC: an endogenous anion channel of the Golgi complex. Biophys $J$ 2000; 78:2918-2928.

13 Van Baelen K, Dode L, Vanoevelen J, et al. The Ca2+/Mn2+ pumps in the Golgi apparatus. Biochim Biophys Acta 2004; 1742:103-112.

14 Madan V, Castello A, Carrasco L. Viroporins from RNA viruses induce caspase-dependent apoptosis. Cell Microbiol 2008; 10:437-451.

15 Jessen F, Sjoholm C, Hoffmann EK. Identification of the anion exchange protein of Ehrlich cells: a kinetic analysis of the inhibitory effects of 4,4'-diisothiocyano-2,2'-stilbene-disulfonic acid (DIDS) and labeling of membrane proteins with $3 \mathrm{H}-$ DIDS. J Membr Biol 1986; 92:195-205.

(Supplementary information is linked to the online version of the paper on the Cell Research website.) 